\author{
Benavent Méndez, Rocío \\ Investigadora UPV DCADHA grupo Animación UPV
}

\title{
Retrospectiva y panorama actual de los Festivales de Animación en España
}

\section{Retrospective and current panorama of the Animation Festivals in Spain}

TIPO DE TRABAJO: Comunicación.

\author{
PALABRAS CLAVE \\ Animación, Festivales, España, Análisis, Cultura, Arte, Industria.
}

KEY WORDS

Animation, Festivals, Spain, Analysis, Culture, Art, Industry.

RESUMEN

Los festivales son eventos que generan comunidad, y por ello, son relevantes tanto para la sociedad como para los artistas que representan esa disciplina artística, porque unen a agentes culturales, sociales y políticos importantes para la continuidad de ese arte en el ámbito público. En nuestro caso, vamos a analizar los festivales de cine de animación que han existido y existen en España, para poder conocer mejor su historia y su tendencia futura de esta disciplina audiovisual.

En este país, como en tantos otros, la animación es considerada una disciplina marginal respecto a la historia del cine pero básica para la constitución del séptimo arte. Así mismo la génesis de los festivales de animación llega en un contexto muy particular y con intenciones de establecerse poco a poco en la memoria colectiva de los amantes de este arte. Esta revisión retrospectiva, pretende conducirnos a una panorámica de la situación actual, analizar las tendencias futuras en cuanto a estos formatos culturales se refiere y dar a conocer las posibilidades existentes a los nuevos creadores, ya que actualmente todavía son grandes desconocidos para los futuros profesionales del sector.

Esta comunicación, toma como base la realización de una búsqueda intensiva de los referentes existentes, permitiéndonos un acercamiento a los diferentes festivales de animación desde su creación y su trayectoria. Empleando una metodología de investigación descriptiva de la trayectoria histórica de los festivales del pasado y del presente, podremos valorar las diferentes manifestaciones artísticas que se dan en los procesos culturales que llevan a la creación y continuidad de los festivales en el tiempo. Esta panorámica pretende abrir la visión de las múltiples posibilidades de formatos que existen y que construyen la identidad de la animación actual española.

\section{ABSTRACT}

Festivals are events that generate community, and therefore, are relevant to society as well as to the artists who represent that artistic discipline, because they unite important cultural, social and political agents for the continuity of that art in the public sphere. In this case, we are going to analyze the animation film festivals that have existed and exist in Spain, in order to better understand its history and future trends in this audiovisual discipline.

In this country, as in others, animation is a marginal discipline with respect to the history of cinema but basic for the constitution of the seventh art. Likewise, the genesis of animation festivals comes in a very particular context and with the intention of establishing little by little in the collective memory of lovers of this art. This retrospective review aims to provide an overview of the current situation, analyze future trends and formats sector. 
This communication, based on the realization of an intensive search of existing relationships, allows an approach to the different festivals of animation from its creation and its trajectory. Using a descriptive research methodology of the historical trajectory of past and present festivals, we will be able to assess the different artist manifestations and cultural processes that lead to the creation and continuity of festivals over time. This panorama aims to open the vision of the multiple possibilities of the formats that exist and that build the identity of the current Spanish animation.

\section{INTRODUCCIÓN}

Los festivales de animación generan comunidad, y por ello, son relevantes tanto para la sociedad como para los artistas que representan esa disciplina artística, porque unen a agentes culturales, sociales y políticos importantes para la continuidad de ese arte en el ámbito público. En nuestro caso, se trata de los festivales de cine de animación que han existido y existen en España desde inicios del Siglo XXI, para poder conocer mejor su historia y su tendencia futura de esta disciplina audiovisual.

A pesar de que existan miles de festivales que contemplen la animación como sección secundaria en sus festivales, queremos señalar los pocos exclusivos de esta disciplina que han existido hasta ahora para poder valorar el panorama actual y las nuevas tendencias futuras de este formato. Son unos festivales muy particulares y con unas idiosincrasias bastante concretas. Vamos a ver los referentes existentes con un tratamiento histórico, pero sobretodo reflexivo de que es lo que significan estos festivales para el sector de la animación española y sus audiencias, abriendo la visión de las múltiples posibilidades de formatos que existen y que construyen la identidad de la animación actual española.

\section{METODOLOGÍA}

La metodología utilizada viene de la búsqueda intensiva de referentes existentes, ya que existe muy poca literatura científica en general sobre el tema, pero la observación participante también nos dan las pautas para esta investigación. Empleamos una metodología de investigación descriptiva de la trayectoria histórica, desde los años noventas hasta la actualidad, haciendo un recorrido de los festivales del pasado y del presente, para poder esbozar los procesos culturales que se llevan a cabo para la creación y continuidad de los festivales de cine de animaicón en el tiempo. El primero en España fue la Muestra de Animac, que se creó en 1996 y ha durado hasta la actualidad.

En esta parte cabe decir, que las investigaciones respecto a los festivales de cine en general "se pone en evidencia la ausencia de textos referidos a España" (Jurado y Nieto, 2014, p.2). Con este panorama desolador es evidente que en realación al cine de animación todavía son más escasos.

Únicamente encontramos textos sobre historia de la animación Nuevo cine de animación en el estado español 2000-2006 (2006) y Breve historia del cine de animación en España (1993) ambos de Emilio de la Rosa, pero nada que tenga que ver con el origen de los festivales de cine de animación en España.

\section{DESARROLLO}

El origen del término "festival" etimológicamente procede de la palabra en inglés "festival" y con ella del latín "festivālis", espectáculos culturales, exposiciones o concursos, jolgorio, socialidad, etc. Cualquier actuación que tiene como fin entretener o distraer, como una ocasión para festejar o celebrar, especialmente un día y a una hora particular, que se repite a intervalos regulares.También puede ser un grupo de representaciones que dedicadas a los artistas o a un arte en particular.

Un festival, en general, es un evento que se celebra en una comunidad y se centra en algún aspecto característico de esa comunidad y su cultura. Los festivales a menudo sirven para cumplir con propósitos comunales específicos de esa comunidad y ecosistema particular. Estas celebraciones ofrecen un sentido de pertenencia para grupos religiosos, sociales o geográficos, lo que contribuye a la cohesión grupal del mismo. También pueden proporcionar entretenimiento, y se centran en temas culturales o étnicos, siendo necesaria la participación de su comunidad para la continuidad de los mismos.

En la antigua Grecia y Roma, los festivales estaban estrechamente relacionados con la organización social y los procesos políticos. En particular, los festivales de cine son los que están mejor considerados según los profesionales del sector para poder generar comunidad y espacios comunes como explican a continuación Jurado y Nieto. 
trabajos son, además de los festivales de cine, las salas de exhibición comercial de aquellos que excepcionalmente proyectan cortos antes de un largometraje, las cadenas de televisión que esporádicamente y/o intermitentemente encuentran hueco en su programación; y fundamentalmente Internet. De todos, sólo los certámenes están considerados los espacios más adecuados (Jurado y Nieto, 2014, p.102).

Todos estos aspectos se ubican hoy en los estudios sobre las industrias culturales y creativas. Los festivales de cine tienen un poder de estimular a las comunidades artísticas, pero sobre todo de hacer visibles obras que de otra manera se hacen bastante imposibles de ver.

La función de poner al alcance del público el cine no comercial es probablemente una de las significativas a nivel cultural, por la alternativa y la innovación que supone en la programación de películas, mostrando nuevas temáticas, creadores, profesionales en ciernes, etc. (Jurado y Cortés, 2018, p.92)

Los certámenes cinematográficos tienen una labor que no tratan otros formatos culturales ni medios, poniendo en valor las creaciones de esas disciplinas, sensibilizándolas y mostrando todo lo que se hace, que de otro modo no se podría exhibir.

El aspecto más interesante [de los festivales] es que acercan una buena oferta cultural a un público que normalmente no tiene acceso a ese tipo de creación audiovisual y suelen incluir retrospectivas interesantes que dan a conocer las obras de autores poco difundidos en los medios. (Mosquera, 2002, p.68)

Estos encuentros son la primera toma de contacto real del creador con su público o del estudiante con su sector. Se trata de un escaparate tanto para los que están empezando, como los que ya están metidos en el mercado, conectando sus cabezas, intereses, currículims, trayectorias para futuras colaboraciones o conexiones aunque sea. "Un festival de cine es primero una fiesta y luego, casi inmediatamente, una instancia de negocios." (Maza, 2008).

Velázquez y Ramírez (2000) citados por Jurado y Cortés (2018) destacan la labor de los festivales como exhibidores (...) "en muchas ocasiones son el único escaparate donde las producciones españolas, tanto de largo como de cortos, verán la luz; Los festivales son, en este momento, la ventana más abierta para la exhibición pública del cortometraje español. Es el espacio natural, y a veces, único, en el que muchos trabajos pueden confrontarse con el público." (p.91)

Es evidente el valor que pueden tener los festivales en ámbitos artísticos, pues son las galerías de arte del audiovisual y los mercadillos de "gangas" del sector cinematográfico. (...) Es por tanto, más realista pensar que el paso por festivales puede ser muy positivo para mostrar en pantalla grande el cortometraje a sus potenciales compradores. Y esta posibilidad se multiplica si el festival es internacional. (Jurado y Cortés, 2018, p.91)

Para poder hablar de los festivales de animación en España nos tenemos que situar a finales de los años noventa, en concreto en 1996. El festival de Annecy en Francia es el más antiguo de los festivales internacionales a nivel mundial y su aparición fue en 1960 de forma bienal,pero en 1996 pasó a ser un festival anual y con un gran futuro por delante.

En España, también surgió el Animac en la misma época, ya que fue creado en 1996 con el objetivo de promocionar a animación audiovisual y ser un punto de encuentro de profesionales de la animación en nuestro país. Con la excusa de celebrar el centenario del cine, Animac comenzó su andadura como muestra que se mantiene en la actualidad. En aquel año, apareció con otro nombre, Cinemagic 96, Festival Internacional de Cine de Animación, bajo la dirección de Eladi Martos y Jordi Artigas. Pero al año siguiente, tomó finalmente el nombre de Animac, Festival Internacional de Cine de Animación. En 2006 ya se estableció como la Mostra Internacional de Cinema d'Animació de Catalunya, organizada y producida por el Ayuntamiento de Lleida y la Generalitat de Catalunya. A lo largo de estos 23 años de historia es la muestra española con más ediciones realizadas y uno de los eventos del audiovisual animado más conocido en el campo de la animación internacional celebrada en España.

Animac tiene una importancia y un gran prestigio a nivel internacional. Siendo una muestra, no un festival, ha conseguido poner en relevancia los trabajos que usan la animación como instrumento para su expresión artística. Animac, actualmente representa el único "festival" de España que funciona como laboratorio de ideas para los nuevos creadores, yendo más de los límites y del tiempo, apoyando las obras independientes que arriesguen y aporten novedades.

También cabe destacar la labor que durante algo más de diez años realizo Animadrid, Festival Internacional de Imagen Animada de Pozuelo de Alarcón-Comunidad de Madrid. Desde el año 2000 hasta el 2011. En 2001, el certamen puso su sede en Pozuelo de Alarcón (Madrid), convirtiéndose desde entonces en un festival internacional de animación, en el que se incluyeron nuevas secciones competitivas de producción extranjera, retrospectivas, presencia de escuelas de cine, homenajes, sesiones para niños y jóvenes, talleres, concursos, etc. Por Animadrid pasaron grandes personalidades de la animación española e internacional, realizadores tan importantes como Ray Harryhausen, María Trenor, Jordi Amorós, Juan José Mendy, Sonia Llera, Pablo Llorens, Juan Pablo Zaramella, entre otros. 
Otros festivales que acabaron desapareciendo con los años o no son tan conocidos, son el caso de: Animabasauri, Festival Internacional de Cine de Animación de Basauri-Bizkaia, que dejó de exitir en 2012 con su 8a Edición; Festival de Cine de Animación AnimaTeruel, que finalizo en 1999; AnimaCaceres, Ani-mar Festival Internacional de Animación, que apenas duró un año, Tenerife Internacional Animation Festival en 2002, Animacor 2003-2011, etc.

\begin{tabular}{|l|l|l|l|}
\hline \multicolumn{1}{|c|}{ Name } & \multicolumn{1}{|c|}{ Est. } & \multicolumn{1}{c|}{ City } \\
\hline $\begin{array}{l}\text { Animac - Catalunya International } \\
\text { Animation Film Festival }\end{array}$ & 1996 & Lleida & $\begin{array}{l}\text { Special } \\
\text { interest }\end{array}$ \\
\hline Festival de Cine de Sitges & 1967 & Sitges & $\begin{array}{l}\text { Special } \\
\text { interest }\end{array}$ \\
\hline Gijón International Film Festival & 1963 & Gijón & International \\
\hline $\begin{array}{l}\text { Las Palmas de Gran Canaria } \\
\text { International Film Festival }\end{array}$ & 2000 & $\begin{array}{l}\text { Las Palmas de Gran } \\
\text { Canaria }\end{array}$ & International \\
\hline Málaga Spanish Film Festival & 1998 & Málaga & Spanish \\
\hline Marbella Film Festival & 2006 & Marbella & International \\
\hline $\begin{array}{l}\text { San Sebastián International Film } \\
\text { Festival }\end{array}$ & 1953 & San Sebastián & International \\
\hline Seminci & 1956 & Valladolid & International \\
\hline
\end{tabular}

Figura I. “Tabla de festivales de animación de Wikipedia”. Fuente (https://en.wikipedia.org/wiki/List_of_film_festivals_in_Europe\#Spain)

A pesar de lo que pueda parecer, y como podemos ver en la tabla, sólo se ha tenido en cuenta a Animac como festival de animación importante en España, junto a él, están otros festivales generalistas que tienen secciones competitivas de animación pero que no son únicamente de esta disciplina. Entre ellos podemos destacar las secciones que apoyan la animación española dentro de estos festivales: Festival de Sitges, Festival de Málaga, Festival de Alicante, Curtas Fest, ABYCINE- Festival Internacional de Cine de Albacete, Rafal en Corto, Festival de cine corto ciudad de Consuegra, Becurt, Aguilar de Campo, Festival Internacional de cine de Gijón, Seminci, Festival de Lanzarote, Festival Ibérico de Cinema de Badajoz, Festival de cortometrajes de Calahorra"iiCORT...en!!, Filmets, iRequena y Acción!, Festival internacional de cine independiente de Elche, Avilacine, Festival Internacional de Cine de Calzada de Calatrava, Festival de Cine de Sant Joan d'Alacant, Medina del Campo Film Festival, Punto y raya festival etc.

A pesar de todo, algunos de los festivales exclusivos de animación activos en España son: Animac (1996), Animalada-Sevilla animation festival (2013), Non Stop Barcelona (2009), Mecal Pro (1998), Animayo: Intenational film festival of animation, visual effects and video games (2006), Animatic Muestra Internacional de Cine de Animaciónde la Fundación Caja Navarra (2007), Imaginaria Huesca (2013), Summa 3D (2013), Animadeba (2008) y el recién nacido Animakon, Bilbao international festival (2017). También existen puntos de encuentro que no son únicamente festivales sino que van más allá de las proyecciones y que pretenden generar mercado y debates sobre la animación en España: 3D Wire (2008), Mundos digitales (2015) y Congreso Quirino (2018). Dónde los jóvenes realizadores pueden hacer pitch para presentar sus cortos y empezar a ser considerados en el mercado laboral.

En particular en la Comunitat Valenciana tenemos secciones o se tiene en consideración la animación en Cinema Jove (1986), Dona y cinema (2014), Quartmetratges (2010), Cortometrajes por la igualdad (2006), La cabina (2008), Festival Internacional de Cine y DDHH Valencia Human Fest (2015) y Catacumba film festival (2002).

Cabe destacar el Prime the Animation (2012) como el único festival internacional de jóvenes talentos de España. Lleva 7 años y es de las posibilidades más novedosas en el panorama actual de la animación en España para dar visibilidad a los nuevos talentos. Si ganan el concurso se les contrata para poder hacer la imagen del festival del año siguiente. Además, encontramos Corto Comenius (2006) o Cinemística-Jóvenes (2014) como festivales para jóvenes realizadores de la animación y vinculados a asociaciones o escuelas. 
Podemos considerar además los premios como un punto de partida. Actualmente hay solo dos que pueden hacer que los cortometrajes se visibilicen más aún: Premios Fugaz de Cortoespaña (2017) y por supuesto Premios Quirino de la animación iberoamericana (2018) que a pesar de su corto recorrido están siendo de gran relevancia internacional.

Finalmente, otra manera de llegar al mercado y no menos importante, son los catálogos de comunidades autónomas como es el caso de CURTS (2008) de la Filmoteca de València o FILNOW (2016) de la Escuela de Cine de Madrid (ECAM) que llevan la distribución gratuitamente a los festivales más interesante para favorecer la visibilidad del producto autonómico y nacional.

\section{CONCLUSIONES}

Todos estos festivales ayudan a los jóvenes talentos a experimentar una posibilidad de conexión y reunión con su comunidad más afín. En el caso de los estudiantes, ellos son el caldo de cultivo para poder hacer frente a las exigencias futuras de la industria y en este tipo de encuentros pueden ser captados por agentes que los necesiten.

Para los animadores, como creadores y profesionales, es una oportunidad de darse a conocer, tanto por su trabajo y talento como por sus habilidades sociales puestas a disposición del festival al que asistan. Ya que a través de ellos se pueden llegar a poner en contacto con productores, distribuidores y profesionales del sector que en un futuro les pueden proveer de trabajos.

En particular, los festivales de cine de animación median entre los agentes culturales y los artistas y está es una de sus funciones más importantes. Generan esa comunidad que crea comunicación específica sobre nuevos proyectos, buenos porfolios o posibles creaciones animadas.

Aunque en general los festivales de cine puedan parecer un negocio del prestigio, se legitiman propiamente al otorgar premios a los filmes, ganando así prestigio el evento, sin embargo, las películas se abren camino en cuanto logran premios o reconocimientos en festivales acreditados. Son los epicentros de las de la industria del cine y en particular, constituyen un lugar importante de alcanzar para los nuevos creadores.

\section{FUENTES REFERENCIALES}

Jurado Martín, M. y Nieto Martín, A. (2014). Nuevas propuestas, viejos circuitos. El papel de los festivales de cine españoles en la consolidación de los nuevos realizadores. Secuencias. Revista Historia del cine, 39 (2014), 100-122.

Jurado Martín, M. y Cortés Selva, Laura (2018). Y, ¿para qué sirven los festivales de cine? Estudio sobre las funciones de los certámenes cinematográficos en España (2000-2002). Sphera Publica, 1 (18), 83-103.

Maza, G. (2008). ¿Para que sirven los festivales de cine?. laFuga, 7 (2008). http://2016.lafuga.cl/para-que-sirven-los-festivales-de$\underline{\text { cine } / 304}$

Mosquera, L. (2002). Corto y Televisión. En Cerón Gómez, J. F. (coord.), Años de corto. Apuntes sobre el cortometraje español desde los noventa. Murcia: Universidad de Murcia y Primavera Cinematográfica de Lorca.

Rosa, E. de la, y Vilar, H. (1993). Breve historia del cine de animación en España. Teruel: AnimaTeruel. 